\title{
Challenges and Opportunities in Atrium Buildings: A Review
}

\author{
Susan Bajracharya \\ Department of Mechanical Engineering, Institute of Engineering, Central Campus, Pulchowk, \\ Tribhuvan University, Nepal \\ Corresponding email: bajracharya.susan@yahoo.com
}

\section{Overview}

One of the most significant problems regarding the space conditioning of atriums is their relatively large volumes compared with traditional commercial and institutional spaces. This may lead to high energy consumption, if atriums are fully conditioned unless effective design strategies are implemented. Most people perceive atriums as indoor environments, not as outdoor or intermediate environments and therefore demand high comfort levels. It is often very difficult to achieve high thermal comfort and low energy consumption at same time. A $1{ }^{\circ} \mathrm{C}$ decrease in indoor temperature in winter will decrease energy consumption by $5 \%$. If people are aware of such relationships, it might lead them to accept atriums with lower temperatures in winter and higher temperature in summer and wear appropriate clothing while in atrium spaces, thus achieving considerable savings in energy use.

\section{Energy conservation design measures}

There are many potential energy conservation measures for energy efficient atrium buildings. The most important are as follows:
1) Buffer zones
2) Optimum orientation and configuration of the atrium building
3) Efficient design and operation of HVAC system
4) Stratified cooling,
5) Passive cooling
6) Passive heating, and
7) Daylighting

\section{1) Energy conservation by creating buffer zones:}

Use of atrium as a buffer zone, which is a transition space between the indoor and outdoor environments, could provide energy savings. Heat transfer through walls is a function of the temperature gradient between the two sides of the wall. Therefore, the heat transfer intermediate walls of spaces facing an atrium may be considerably reduced compared with exterior walls, even with the high U-values of intermediate walls. This could be achieved by maintaining the temperature of the atrium slightly higher in summer and lower in winter than that in the adjacent occupied space of building and keeping the ration of exposed surface to interior surface at its lowest. In a core type atrium with cubic shape, one surface shields four interior surfaces of equal 
area. The buffering effect of atriums is beneficial whether the occupied spaces are being heated or cooled, because it reduces heat gain and heat loss during summer and winter respectively. Atriums also protect walls of the buildings facing them from direct solar radiation, rain and infiltration caused by wind.

\section{2) Energy conservation by Optimum orientation and configuration of the atrium building}

Orientation of glazed surfaces is one of the most important design considerations for energy conservation in atrium spaces. The impact, however, is far more important for linear atriums than square atriums. Furthermore, sensitivity to orientation and slope decreases as U-value and visible transmission decrease. As it is difficult to control low-angle solar radiation, it is better to avoid east- or west oriented glazed walls; north- or south-oriented atriums are preferable. In an internal load dominated building in a warm climate, a glazed wall facing north is useful to avoid solar gain, while preserving a glare-free view. In cool climates, a south-oriented atrium wall is the most useful, particularly if it is designed to keep out high-angled summer solar radiation while admitting low-angled winter solar radiation for passive solar heating.

Configuration of atriums is another important design factor for energy conservation, whether unconditioned, partially conditioned or fully conditioned. For attached and linear atrium buildings with equal area, the former will be more energy-conserving, it he atrium is unconditioned, while the latter will be more energy-conserving if the atrium is partially of fully conditioned, because of the large proportion of exterior glazed surfaces exposed to outdoors in attached atriums. Likewise, core-type atriums will be more appropriate if full conditioning is required, because less glazed surface is exposed to outdoors than in envelope type. Furthermore, the temperature swing is likely to be more profound in attached atriums than in linear atriums with the same area, because of the increased ratio of glazed to intermediate surfaces. Atriums with high sectional aspect ratio are subjected to greater temperature stratification causing high stack effect, while low sectional aspect ratio and high plan aspect ratio are more appropriate for daylighting, passive heating and radiative cooling.

\section{3) Energy conservation by efficient operation and design of HVAC systems}

Use of atriums as buffer zones with passive cooling and heating is the most energy conserving strategy. However, if partial or full conditioning is required, it is still possible to build energy efficient atrium buildings through more efficient operation and design of HVAC systems.

Studies have shown that implementation of efficient operation techniques suchas thermostatic control (night set-back in heating mode and night set-up in cooling mode, increase in dead band) and destratification help to reduce energy consumption in atrium buildings. Furthermore, efficient design of HVAC system with variable air volume system instead of constant air volume system and heat pump type system instead of electrical heating system reduces energy consumption.

Incorporation of thermal storage techniques in conjunction with intelligent building automation system is another viable option for energy conservation, which might help to reduce peak load demand. 


\section{4) Energy conservation by stratified cooling:}

In conventional total volume cooling systems, the temperature of air throughout an entire space will be maintained close to the same level. In air mixing cooling systems (as opposed to displacement ventilation systems), cool supply air will usually be distributed from the top level and return air will be collected either at the bottom or top level. If the occupied zone is only up to a few meters above floor level, not throughout the entire volume. In such cases, a stratified cooling system could be used successfully.

Gorton andSassi conducted extensive study on stratified cooling, which is a techniqueof cooling only the lower, occupied zone of high-ceiling space. Their study showed that thermally stratified cooling system may be especially helpful in reducing some potential thermal loads by isolating them in the upper zone, thus reducing the initial and operating costs of the equipment compared with a conventional total volume cooling system for the same space.

\section{5) Energy conservation by passive cooling}

As atrium buildings have extensive and are usually used during daytime, they may require cooling for the majority of annual operating hours even in cold climates. Passive cooling techniques that can be used in atriums are as follows:
a) Control of solar heat gain (shading)
b) Use of thermal mass
c) Radiative cooling, and
d) convective cooling

Solar shading devices may reduce solar heat gain in summer. Vertical exterior shading are most effective on the east and west fot lower sun angles, horizontal on the south for higher sun angles. However, fixed shading devices may reduce solar heat gain as well during and may have a negative effect overall. Therefore, detail calculation must be carried out for each case before utilizing such technique. Variable external shading might be the solution, which provides desired effect in both summer and winterThermal mass of the building can help to reduce the temperature of the building at and ultimately absorb the heat generated in building during the daytime. This concept works well in climates where night temperature drop below $20{ }^{\circ} \mathrm{C}$ and diurnal temperature swing is $10{ }^{\circ} \mathrm{C}$. At night, the cold air from outside can be used to flush the heat absorbed in an atrium during daytime and to cool the bed rock bed. During the daytime cool air from rock beds may be supplied from the bottom of the atrium.

The cold night sky and the polar sky during mild summer days can serve as a heat sink for the radiative cooling of a building. Heat will transfer most effectively by means of radiation from a warm atrium to the cooler sky, if the sky is clear. The potential for radiative cooling decreases as the sky becomes cloudy or humid.

Convective cooling (natural ventilation) is mainly based upon the stack effect, which increases as Sectional Aspect Ratio (SAR) increase and Plan Aspect Ratio (PAR) and Aspect Ratio (AR) decrease. Natural ventilation driven by stack effect might be capable of maintaining comfort conditions in regions with summers. One of the most important conditions for natural ventilation is that the temperature of outdoor air should be less than indoor air. 


\section{6) Energy conservation by Passive heating}

In most commercial, industrial and office buildings, perimeter heating is not as significant concern as lighting and cooling, because environmental control in these buildings is usually dominated by internal heat gains and ventilation requirements. Heating may be required in residential buildings, hotels or museums. Many internal load dominated buildings sometimes require core-cooling and perimeter-heating.

In winter, large glazed areas help to warm an atrium by admitting large amounts of solar radiation, and massive concrete walls and floors can store the solar heat. As it is a property of glazed materials to admit solar radiation with short wavelengths and trap radiation with long wavelengths, it is possible to store large amounts of heat inside atriums. Furthermore, if that heat is stored in some kind of thermal mass, it could be recirculated into atriums in order to avoid condensation during cold nights or directed to other spaces requiring heat during the day. The problems associated with passive are over-heating, radiation loss, and down drafts. Temperatures inside atriums may rise far above comfort levels during sunny days. During overcast days and nights, atrium in cold regions may experience radiative heat loss as well as down drafts. The potential for utilization of direct solar radiation depends upon the proximity to taller buildings and orientation of the building, which determine the availability of direct solar radiation.

\section{7) Energy conservation by daylighting}

Studies have shown that energy consumption by artificial lighting in Europe is about $50 \%$ of total electrical energy consumed in commercial, institution and office buildings. While in North American countries, it accounts for only $20 \%$. Therefore, daylighting is one of the potential areas to reduce building energy use as well as peak energy demand. The daylighting potential of atriums depends upon their ability to admit daylight to adjacent spaces.

There is a wide range of glazing materials available to control solar transmission, and U-values. One disadvantage is that is affects the whole year. Dark colors might give a continuous dull impression. Large glazed area in atriums will help to admit more daylight into the centre of a building and allow reduction in the use of artificial lighting. Artificial lighting not only consumes electrical energy but also produces a large amount of heat, which eventually increases cooling loads in hot weather, but may decrease heating loads in winter. For each case, it is necessary to determine the optimal balance between daylighting, cooling and heating loads in a way that ultimately reduces total annual energy use. 